OPEN ACCESS

Edited by:

In-Kyu Lee,

Kyungpook National University,

South Korea

Reviewed by:

Yu Pan

Shanghai Ninth People's Hospital,

China

Jin Joo Cha,

Korea University, South Korea

${ }^{*}$ Correspondence: Huijie Zhang

huijiezhang2005@126.com Yaoming Xue

yaomingxue@126.com

${ }^{t}$ These authors have contributed equally to this work and share

first authorship

Specialty section:

This article was submitted to

Renal Endocrinology,

a section of the journal

Frontiers in Endocrinology

Received: 07 September 2021 Accepted: 12 October 2021 Published: 28 October 2021

Citation:

Huang Y, Liu W, Liu J, Guo D,

Zhang P, Liu D, Lin J, Yang L, Zhang H

and Xue $Y$ (2021) Association of

Urinary Sodium Excretion and

Diabetic Kidney Disease in Patients

With Type 2 Diabetes Mellitus:

A Cross-Sectional Study.

Front. Endocrinol. 12:772073.

doi: 10.3389/fendo.2021.772073

\section{Association of Urinary Sodium Excretion and Diabetic Kidney Disease in Patients With Type 2 Diabetes Mellitus: A Cross-Sectional Study}

\author{
Yan Huang ${ }^{1 \dagger}$, Wenhui Liu ${ }^{1 \dagger}$, Jianfang Liu ${ }^{1+}$, Dan Guo ${ }^{1}$, Peizhen Zhang ${ }^{1}$, Deying Liu ${ }^{1}$, \\ Jiayang Lin ${ }^{1}$, Linjie Yang ${ }^{1}$, Huijie Zhang ${ }^{1,2,3^{*}}$ and Yaoming Xue ${ }^{1 *}$ \\ ${ }^{1}$ Department of Endocrinology and Metabolism, Nanfang Hospital, Southern Medical University, Guangzhou, China, \\ ${ }^{2}$ Guangdong Provincial Key Laboratory of Shock and Microcirculation, Guangzhou, China, ${ }^{3}$ Department of Food Safety and \\ Health Research Center, School of Public Health, Southern Medical University, Guangzhou, China
}

Background: Diabetic kidney disease (DKD) is the leading cause of end-stage kidney disease worldwide. Epidemiological evidence of the association between urinary sodium excretion and the presence of DKD in patients with type 2 diabetes mellitus (T2DM) has not yet been well established.

Methods: We performed a cross-sectional study of 1545 patients with T2DM over aged 20 years old from January 2018 to December 2020. Urinary sodium excretion was measured by 24-hour urine samples in inpatients and morning fasting urine samples in outpatients. The associations between urinary sodium excretion and the risks of DKD were examined using stepwise regression analysis, logistic regression analysis and multivariable-adjusted restricted cubic splines (RCS).

Results: Regression analysis showed that urinary sodium was independently associated with urinary albumin to creatinine ratio (UACR) level $(P=0.006)$ and the risks of DKD $(P=0.042)$. In multivariable-adjusted RCS analysis, urinary sodium excretion was significantly associated with UACR in all patients $(P=0.008)$, and exhibited a J-shaped relationship. Logistic regression analysis showed that increased urinary sodium excretion was significantly associated with increased risks of DKD [OR (95\% Cl); 1.56 (1.07-2.27); $P=0.020]$. However, the relationships between urinary sodium excretion and the risks of DKD and albuminuria showed no significance, after further adjustment for HOMA-IR and ba-PWV (brachial-ankle pulse wave velocity) (Both $P>0.05$ ).

Conclusions: Higher urinary sodium excretion level was associated with increased risks of DKD among patients with T2DM, dependent of vascular sclerosis and insulin resistance.

Keywords: diabetic kidney disease, type 2 diabetes, urinary sodium excretion, insulin resistance, vascular sclerosis 


\section{INTRODUCTION}

Diabetic kidney disease (DKD), one of the common complications of diabetes mellitus, was strongly associated with all-cause and cardiovascular disease (CVD) mortality in a multiethnic Asian population (1). DKD is the leading cause of end-stage kidney disease worldwide, accounting for approximately $50 \%$ of cases in the developed countries (2). The World Health Organization (WHO) multinational study showed that renal disease including DKD accounted for $11 \%$ of all deaths of patients with type 2 diabetes mellitus (T2DM) (3). In China, approximately 24.3 million have DKD and $60.5 \%$ of patients with diabetes have reduced kidney function or slightly increased albuminuria (4).

It has been well documented that sodium intake is positively associated with the risks of clinical CVD events and chronic kidney disease (CKD) $(5,6)$. However, the association between sodium intake and the risks of DKD remains less clear. Albuminuria has emerged as a sensitive marker of kidney damage (7), and a predictive risk factor for end-stage renal failure in diabetic individuals (8). Epidemiological evidence conducted in the general population and patients with type 1 diabetes and reported that sodium intake was positively related to urinary albumin excretion $(9,10)$, particularly in overweight subjects (10). Additionally, few studies reported a reverse J-shaped or no significant association between dietary sodium and urinary albumin in patients with T2DM $(11,12)$. Indeed, dietary sodium intake measurement methods may contribute to these conflicting findings. However, these evidences did not provide a conclusive information regarding the association between sodium intake and DKD in patients with T2DM. We quantified urinary sodium excretion by 24-hour urine samples, which be considered the most reliable estimate of sodium intake (13), and aimed to explore the associations between urinary sodium excretion and the risks of DKD in patients with T2DM.

\section{MATERIALS AND METHODS}

\section{Study Participants}

This cross-sectional study was based on the Nanfang Prospective Diabetes Study (NFPDS), a prospective cohort study designed to explore the associations of urinary electrolyte and possible risk factors of microvascular complications in patients with T2DM from Nanfang Hospital, Southern Medical University. We followed the methods of Liu et al. (14). A total of 1545 inpatients and outpatients over aged 20 years old from Guangzhou city, China, was included in this study from January 2018 to December 2020. In the present study, participants completed urine collections and evaluation of diabetic microvascular complications. Inclusion criteria was diagnosis of T2DM according to the criteria of the American Diabetes Association (ADA) (2017) (15). The individuals undergoing dialysis treatment, being pregnant or planning to become pregnant, and patients with NYHA class III or IV congestive heart failure and severe systemic infection were excluded. All participants completed a uniform questionnaire regarding demographics, lifestyle habits (i.e., smoking status, alcohol consumption) and medical history.

The protocol for the study received ethical approval conforming to the Declaration of Helsinki from the Institutional Review Board of Nanfang Hospital, Southern Medical University. All participants provided written informed consent.

\section{Measurements}

Physical examination included height, weight and blood pressure (BP) were screened following a standardized protocol. The height and weight were measured by using the same automatic measuring instrument. Body mass index (BMI) was calculated as weight $(\mathrm{kg})$ divided by square of height $\left(\mathrm{m}^{2}\right)$. BP was measured in triplicate with an electronic sphygmomanometer (OMRON Company). The mean value of the three readings were used for analysis. ba-PWV (brachial-ankle pulse wave velocity, ba-PWV) was measured using an arteriosclerosis detection device (Omron, BP-203RPEIII).

Overnight fasting blood samples of inpatients and outpatients were obtained and tested in the laboratory of Nanfang hospital with stringent quality control. Triglyceride (TG), total Cholesterol (TC), low-density lipoprotein cholesterol (LDL-c), creatinine (CR), uric acid (UA) was determined by enzymatic methods using a fully automated biochemical analyzer. Urinary creatinine (Ucr), urinary albumin to creatinine ratio (UACR) were determined by automatic protein - specific analyzer (Afinion AS100). Glycated hemoglobin (HbA1c) was determined by high performance liquid chromatography. Fasting plasma glucose concentrations were determined by the hexokinase method. Fasting insulin level was determined by electroluminescent immunoassay. According to the test results, index of homeostasis model assessment of insulin resistance (HOMA-IR) was calculated according to the following formula: HOMA-IR = fasting insulin $(\mathrm{mIU} / \mathrm{L}) \times \mathrm{FBG}(\mathrm{mmol} / \mathrm{L}) /$ 22.5. Inpatients were asked to collect 24-hour urine samples for the measurement of urinary sodium level. Outpatients were asked to collect morning fasting urine samples to measure spot urinary sodium. The Kawasaki formula was used to estimate 24-hour urinary sodium excretion of outpatients, which be considered valid for estimating sodium intake in healthy participants and patients with antihypertensive therapy $(16,17)$. 24-hour and spot urinary sodium concentrations were measured by ion selective electrode method.

On the basis of the mean of three seated, hypertension was defined as mean BP of 140/90 $\mathrm{mmHg}$ or greater and/or the selfreported use of antihypertensive medication. Hyperlipidemia was defined as total cholesterol (TC) $\geq 6.22 \mathrm{mmol} / \mathrm{l}$, or lowdensity lipoprotein cholesterol (LDL-c) $\geq 4.14 \mathrm{mmol} / \mathrm{l}$, or triglycerides $(\mathrm{TG}) \geq 2.26 \mathrm{mmol} / \mathrm{l}$ and/or self-reported use of lipid-lowering drugs.

\section{Definitions of Albuminuria and DKD}

Albuminuria was diagnosed as UACR $\geq 30 \mathrm{mg} / \mathrm{g}$, while excluding infection and other factors. The value of UACR was obtained by calculating urinary albumin $(\mathrm{mg})$ to creatinine $(\mathrm{g})$ ratio. DKD was diagnosed as UACR $\geq 30 \mathrm{mg} / \mathrm{g}$ and/or eGFR $<60$ 
$\mathrm{ml} \cdot \mathrm{min}^{-1} \cdot\left(1.73 \mathrm{~m}^{2}\right)^{-1}$, while excluding other causes of chronic kidney disease. The estimated glomerular filtration rate (eGFR) was calculated by using the CKD-EPI formula (18). Definitions as described above were obtained according to the criteria of ADA (2017) (19).

\section{Statistical Analysis}

Baseline characteristics were described as means \pm standard deviation (SD), median (interquartile range), frequencies, or percentage. Data that were not normally distributed were logarithmically transformed before analysis. General linear models (GLM) and the chi-square test were used to compare the differences between the four quartiles of urinary sodium excretion. The stepwise regression was used to determine the relationship between variables and DKD. Multivariate logistics regression model was used to estimate relationship between urinary sodium excretion level and the risk of DKD as well as albuminuria. RCS model was used to investigate the relationship between urinary sodium excretion and UACR level. Forest plot was used to examine the relationship between urinary sodium excretion levels and the risk of DKD in different subgroups. According to the recommendations of WHO (20), urinary sodium excretion of less than $2 \mathrm{~g} / \mathrm{d}$ was selected as the reference group for all spline plots. Statistical analyses were performed by using SAS version 9.4 (SAS Institute Inc). A two-sided $P<0.05$ was considered statistically significant.

\section{RESULTS}

Table 1 presents the clinical characteristics of patients categorized by presence of DKD. The mean age of the subjects with DKD was $56.5 \pm 10.6$ years. Subjects with DKD had higher urinary sodium excretion level than those with non-DKD $(3.42 \pm$ $1.48 \mathrm{~g} / \mathrm{d} v s .3 .23 \pm 1.42 \mathrm{~g} / \mathrm{d}$, respectively, $P=0.029)$. Subjects with $\mathrm{DKD}$ exhibited greater age, longer duration of diabetes, higher levels of SBP, DBP, PWV, TG, CR, UA, UACR than those with non-DKD (All $P<0.05$ ). Likewise, The DKD group had higher prevalence of hyperlipidemia and hypertension than the nonDKD group. Furthermore, patients with DKD had higher percentage of using RAS blocking agents, diuretics, statin, SGLT 2i (All $P<0.05$ ). The level of eGFR was lower in patients with DKD compared to those with non-DKD $(P<0.001)$. There were no significant differences in the levels of BMI, HbA1c, glucose, HOMA-IR, TC and LDL-c between two groups (All $P>0.05$ ).

The baseline characteristics of participants categorized by quartile of urinary sodium excretion are showed in Table 2. The median of duration of diabetes was 7 (2-12) years. Patients in the higher quartiles had higher prevalence of smoking, longer duration of diabetes and higher percentage of using antidiabetic medication than those in the lower quartiles (All $P<0.05$ ). In addition, there was a significant increasing trend in the levels of BMI, HOMA-IR and eGFR with increasing urinary sodium excretion. Conversely, there was a significant decreasing trend in the levels of HbA1c, TC and LDL-c with increasing urinary sodium excretion. Levels of SBP, DBP, PWV, TG, UR, UA and
TABLE 1 | Characteristics of patients categorized by presence of DKD.

\begin{tabular}{|c|c|c|c|}
\hline Variables & non-DKD & DKD & $P$-value \\
\hline Sample size & 1209 & 336 & - \\
\hline Urinary sodium (g/d) & $3.23 \pm 1.42$ & $3.42 \pm 1.48$ & 0.029 \\
\hline Age (years) & $54.5 \pm 11.0$ & $56.5 \pm 10.6$ & 0.004 \\
\hline Gender (Male n, \%) & 772 (63.9) & $219(65.2)$ & 0.654 \\
\hline Smoking (n, \%) & $545(45.1)$ & $167(50.2)$ & 0.103 \\
\hline Alcohol use (n, \%) & $398(33.0)$ & $126(37.8)$ & 0.095 \\
\hline Duration of diabetes (years) & $6(2-11)$ & $11(5-16)$ & $<0.001$ \\
\hline $\mathrm{BMI}\left(\mathrm{kg} / \mathrm{m}^{2}\right)$ & $24.5 \pm 3.5$ & $24.7 \pm 3.8$ & 0.290 \\
\hline $\mathrm{SBP}(\mathrm{mmHg})$ & $125.8 \pm 17.5$ & $133.4 \pm 19.4$ & $<0.001$ \\
\hline $\mathrm{DBP}(\mathrm{mmHg})$ & $76.7 \pm 11.0$ & $79.2 \pm 10.7$ & $<0.001$ \\
\hline Hypertension (n, \%) & 353 (29.2) & $184(54.8)$ & $<0.001$ \\
\hline Hyperlipidemia (n, \%) & $652(53.9)$ & $246(73.2)$ & $<0.001$ \\
\hline Antidiabetic medication (n, \%) & $847(70.1)$ & $284(84.5)$ & $<0.001$ \\
\hline RAS blocking agents (n, \%) & $133(11.0)$ & $107(31.9)$ & $<0.001$ \\
\hline Diuretics (n, \%) & $31(2.6)$ & $17(5.1)$ & 0.020 \\
\hline Statin (n, \%) & $132(10.9)$ & $76(22.6)$ & $<0.001$ \\
\hline SGLT 2i (n, \%) & $19(1.6)$ & $13(3.9)$ & 0.009 \\
\hline $\mathrm{PWV}(\mathrm{cm} / \mathrm{s})$ & $1571.6 \pm 311.9$ & $1736.4 \pm 346.9$ & $<0.001$ \\
\hline $\mathrm{HbA1c}(\%)$ & $9.3 \pm 2.6$ & $9.4 \pm 2.4$ & 0.579 \\
\hline Glucose (mmol/L) & $8.7 \pm 4.4$ & $8.7 \pm 5.1$ & 0.876 \\
\hline HOMA-IR & $0.70 \pm 1.01$ & $0.73 \pm 1.18$ & 0.667 \\
\hline $\mathrm{TG}(\mathrm{mmol} / \mathrm{l})$ & $1.44(0.98-2.26)$ & 1.65 (1.08-3.05) & 0.003 \\
\hline $\mathrm{TC}(\mathrm{mmol} / \mathrm{l})$ & $5.00 \pm 1.30$ & $5.07 \pm 1.58$ & 0.381 \\
\hline LDL-c (mmol/l) & $3.21 \pm 0.94$ & $3.23 \pm 0.97$ & 0.712 \\
\hline $\mathrm{CR}(\mu \mathrm{mol} / \mathrm{l})$ & $66.0(55.0-78.0)$ & 80.5 (60.0-111.0) & $<0.001$ \\
\hline UA ( $\mu \mathrm{mol} / \mathrm{l})$ & $354.16 \pm 110.10$ & $377.63 \pm 125.79$ & $<0.001$ \\
\hline UACR (mg/mmol) & $1.1(0.7-2.6)$ & $9.4(3.3-46.0)$ & $<0.001$ \\
\hline eGFR $\left(\mathrm{ml} / \mathrm{min} / 1.73 \mathrm{~m}^{2}\right)$ & $95.83 \pm 22.47$ & $79.52 \pm 31.97$ & $<0.001$ \\
\hline
\end{tabular}

$B M I$, body mass index; SBP, systolic blood pressure; $D B P$, diastolic blood pressure; $R A S$, renin-angiotensin system; PWV, pulse wave velocity; $H b A 1 c$, glycated hemoglobin; HOMA-IR, homeostasis model assessment of insulin resistance; TG, triglyceride; TC, total cholesterol; $L D L-C$, low-density lipoprotein cholesterol; $C R$, creatinine; UA, uric acid; UACR, urinary albumin to creatinine ratio; eGFR, estimated glomerular filtration rat.

UACR, glucose showed no differences among the four quartiles of urinary sodium excretion (All $P>0.05$ ). Of note, the prevalence of $\mathrm{DKD}$ from the lowest quartile to the highest quartile was $19.7 \%, 20.0 \%, 21.0 \%, 26.4 \%$, respectively $(P=0.028)$, adjusted for age and gender.

As shown in Table 3, the levels of PWV, HbAlc, CR, UA and urinary sodium were independently correlated with UACR level and the presence of DKD in stepwise regression analysis (All $P<0.05$ ). In addition, age, gender, and the levels of BMI, DBP, SBP, TC were significantly correlated with UACR level.

The RCS analysis showed that urinary sodium excretion was significantly associated with UACR level in all patients $(P=0.008)$ and males $(P=0.017)$, after adjusted for age, gender, BMI, smoking, alcohol consumption, DBP, HbA1c, use of RAS blocking agents, diuretics, hyperlipidemia, statin, and antidiabetic drugs (Figure 1). However, there was insignificant nonlinear association between urinary sodium excretion and UACR level $(P=0.081)$. A J-shaped relationship was observed between urinary sodium excretion and UACR level.

The multivariable-adjusted odds ratios (ORs) for the association between urinary sodium excretion and the risks of DKD and albuminuria are shown in Table 4. After adjustment for age, gender, BMI, smoking, alcohol consumption, DBP, $\mathrm{HbA} 1 \mathrm{c}$, and use of RAS blocking agents, diuretics, hyperlipidemia, statin and antidiabetic medication, individuals 
TABLE 2 | Characteristics of patients categorized by quartile of urinary sodium excretion level.

\begin{tabular}{|c|c|c|c|c|c|c|}
\hline \multirow[t]{2}{*}{ Variables } & \multirow[t]{2}{*}{ Total } & \multicolumn{4}{|c|}{ Estimated 24-hour urinary sodium excretion level } & \multirow[t]{2}{*}{$P$-value } \\
\hline & & Quartile 1 & Quartile 2 & Quartile 3 & Quartile 4 & \\
\hline Sample size & 1545 & 386 & 386 & 386 & 387 & - \\
\hline Urinary sodium (g/d) & $3.27 \pm 1.44$ & $1.69 \pm 0.45$ & $2.72 \pm 0.25^{b}$ & $3.51 \pm 0.25^{b}$ & $5.16 \pm 1.21^{\mathrm{b}}$ & $<0.001$ \\
\hline Age (years) & $54.9 \pm 10.9$ & $54.8 \pm 11.6$ & $55.5 \pm 10.7$ & $55.4 \pm 10.7$ & $54.0 \pm 10.6$ & 0.226 \\
\hline Gender (Male n, \%) & $991(64.1)$ & $218(56.5)$ & $236(61.1)$ & $257(66.6)$ & $280(72.4)$ & $<0.001$ \\
\hline Smoking (n, \%) & $712(46.2)$ & $161(41.7)$ & $166(43.1)$ & $188(49.0)$ & $197(51.0)$ & 0.024 \\
\hline Alcohol use (n, \%) & $524(34.0)$ & $116(30.1)$ & $127(33.0)$ & $144(37.5)$ & $137(35.5)$ & 0.149 \\
\hline Duration of diabetes (years) & $7(2-12)$ & $4(2-10)$ & $7(2-12)^{b}$ & $8(2-13)^{b}$ & $7(2-13)^{b}$ & $<0.001$ \\
\hline $\mathrm{BMl}\left(\mathrm{kg} / \mathrm{m}^{2}\right)$ & $24.5 \pm 3.5$ & $23.9 \pm 3.5$ & $24.4 \pm 3.5^{\mathrm{a}}$ & $24.5 \pm 3.1^{\mathrm{a}}$ & $25.4 \pm 3.7^{\mathrm{b}}$ & $<0.001$ \\
\hline $\mathrm{SBP}(\mathrm{mmHg})$ & $127.4 \pm 18.3$ & $126.4 \pm 18.4$ & $126.6 \pm 18.6$ & $127.9 \pm 18.6$ & $128.9 \pm 17.4$ & 0.174 \\
\hline $\mathrm{DBP}(\mathrm{mmHg})$ & $77.2 \pm 11.0$ & $77.0 \pm 11.7$ & $76.9 \pm 10.5$ & $76.7 \pm 10.7$ & $78.4 \pm 10.9$ & 0.127 \\
\hline Hypertension (n, \%) & 537 (34.8) & $152(39.4)$ & $131(33.9)$ & $123(31.9)$ & 131 (33.9) & 0.150 \\
\hline Hyperlipidemia (n, \%) & $898(58.1)$ & $227(58.8)$ & $226(58.6)$ & $228(59.1)$ & $217(56.1)$ & 0.822 \\
\hline Antidiabetic medication (n, \%) & $1131(73.2)$ & $238(61.7)$ & $283(73.3)$ & $306(79.3)$ & $304(78.6)$ & $<0.001$ \\
\hline RAS blocking agents (n, \%) & $240(15.5)$ & $61(15.8)$ & $56(14.5)$ & $67(17.4)$ & $56(14.5)$ & 0.650 \\
\hline Diuretics (n, \%) & $48(3.1)$ & $18(4.7)$ & $10(2.6)$ & $9(2.3)$ & $11(2.8)$ & 0.230 \\
\hline Statin (n, \%) & 208 (13.5) & $46(11.9)$ & $52(13.5)$ & $57(14.8)$ & $53(13.7)$ & 0.712 \\
\hline SGLT 2i (n, \%) & $32(2.1)$ & $4(1.0)$ & $10(2.6)$ & $7(1.8)$ & $11(2.8)$ & 0.282 \\
\hline PWV (cm/s) & $1607.1 \pm 326.8$ & $1602.0 \pm 333.3$ & $1602.7 \pm 335.6$ & $1602.7 \pm 319.9$ & $1621.3 \pm 319.0$ & 0.825 \\
\hline $\mathrm{HbA1c}(\%)$ & $9.4 \pm 2.5$ & $10.1 \pm 2.8$ & $9.5 \pm 2.5^{\mathrm{b}}$ & $8.9 \pm 2.4^{b}$ & $8.9 \pm 2.2^{b}$ & $<0.001$ \\
\hline Glucose (mmol/L) & $8.7 \pm 4.6$ & $8.9 \pm 5.4$ & $8.8 \pm 4.5$ & $8.3 \pm 3.9$ & $8.6 \pm 4.3$ & 0.278 \\
\hline HOMA-IR & $0.71 \pm 1.04$ & $0.46 \pm 1.07$ & $0.65 \pm 1.07^{\mathrm{a}}$ & $0.74 \pm 1.04^{\mathrm{b}}$ & $0.99 \pm 0.93^{b}$ & $<0.001$ \\
\hline $\mathrm{TG}(\mathrm{mmol} / \mathrm{l})$ & $1.48(1.01-2.38)$ & $1.38(0.95-2.23)$ & $1.52(0.98-2.42)$ & $1.47(1.02-2.40)$ & $1.58(1.04-2.66)$ & 0.427 \\
\hline $\mathrm{TC}(\mathrm{mmol} / \mathrm{l})$ & $5.01 \pm 1.37$ & $5.08 \pm 1.57$ & $5.14 \pm 1.32$ & $4.86 \pm 1.26^{a}$ & $4.98 \pm 1.29$ & 0.023 \\
\hline LDL-c (mmol/l) & $3.21 \pm 0.95$ & $3.25 \pm 1.02$ & $3.33 \pm 0.97$ & $3.13 \pm 0.95$ & $3.15 \pm 0.82$ & 0.013 \\
\hline $\mathrm{CR}(\mu \mathrm{mol} / \mathrm{l})$ & $68.0(55.0-83.0)$ & $65.0(54.0-84.0)$ & $68.0(55.0-85.0)$ & $70.0(59.0-85.0)$ & $68.0(55.0-80.0)$ & 0.708 \\
\hline $\mathrm{UA}(\mu \mathrm{mol} / \mathrm{l})$ & $359.26 \pm 114.06$ & $354.04 \pm 115.65$ & $356.31 \pm 110.54$ & $364.38 \pm 121.77$ & $362.31 \pm 107.95$ & 0.548 \\
\hline UACR (mg/mmol) & $1.5(0.7-5.3)$ & $1.6(0.8-5.0)$ & $1.4(0.7-6.3)$ & $1.4(0.7-4.3)$ & $1.5(0.8-6.5)$ & 0.264 \\
\hline eGFR $\left(\mathrm{ml} / \mathrm{min} / 1.73 \mathrm{~m}^{2}\right)$ & $92.28 \pm 25.73$ & $91.88 \pm 26.61$ & $91.60 \pm 25.86$ & $90.06 \pm 26.36$ & $95.58 \pm 23.78^{a}$ & 0.022 \\
\hline Diabetic kidney disease $(n, \%)^{c}$ & $336(21.8)$ & $76(19.7)$ & $77(20.0)$ & $81(21.0)$ & $102(26.4)^{\mathrm{a}}$ & 0.028 \\
\hline
\end{tabular}

BMI, body mass index; SBP, systolic blood pressure; DBP, diastolic blood pressure; RAS, renin-angiotensin system; PWV, pulse wave velocity; HbA1C, glycated hemoglobin; HOMA-IR, homeostasis model assessment of insulin resistance; TG, triglyceride; TC, total cholesterol; LDL-C, low-density lipoprotein cholesterol; CR, creatinine; UA, uric acid; UACR, urinary albumin to creatinine ratio; eGFR, estimated glomerular filtration rate.

${ }^{a} P<0.05$ compared with Quartile 1 of urinary sodium.

${ }^{b} P<0.01$ compared with Quartile 1 of urinary sodium.

${ }^{c}$ Adjusted for age and gender.

TABLE 3 | Stepwise regression analysis with UACR level and DKD.

\begin{tabular}{|c|c|c|c|c|c|c|}
\hline Variables & \multicolumn{3}{|c|}{ UACR } & \multicolumn{3}{|c|}{ DKD } \\
\hline Gender & -0.440 & 0.091 & $<0.001$ & - & - & - \\
\hline Smoking & - & - & - & - & - & - \\
\hline Alcohol use & - & - & - & - & - & - \\
\hline $\mathrm{BMI}\left(\mathrm{kg} / \mathrm{m}^{2}\right)$ & -0.019 & 0.012 & 0.098 & - & - & - \\
\hline $\mathrm{PWV}(\mathrm{cm} / \mathrm{s})$ & 0.001 & 0.0002 & $<0.001$ & 0.001 & 0.0002 & $<0.001$ \\
\hline $\mathrm{HbA1c}(\%)$ & 0.110 & 0.016 & $<0.001$ & 0.086 & 0.029 & 0.015 \\
\hline HOMA-IR & - & - & - & - & - & - \\
\hline $\mathrm{TC}(\mathrm{mmol} / \mathrm{l})$ & 0.125 & 0.029 & $<0.001$ & - & - & - \\
\hline LDL-c (mmol/l) & - & - & - & - & - & - \\
\hline $\mathrm{CR}(\mu \mathrm{mol} / \mathrm{l})$ & 1.032 & 0.093 & $<0.001$ & 0.979 & 0.145 & $<0.001$ \\
\hline
\end{tabular}

UACR, urinary albumin to creatinine ratio; DKD, diabetic kidney disease; BMI, body mass index; SBP, systolic blood pressure; DBP, diastolic blood pressure; PWV, pulse wave velocity; HbA1C, glycated hemoglobin; HOMA-IR, homeostasis model assessment of insulin resistance; TC, total cholesterol; LDL-C, Iow-density lipoprotein cholesterol; CR, creatinine; UA, uric acid.

The forward stepwise regression analysis was used to obtain the determinants of UACR and DKD. 

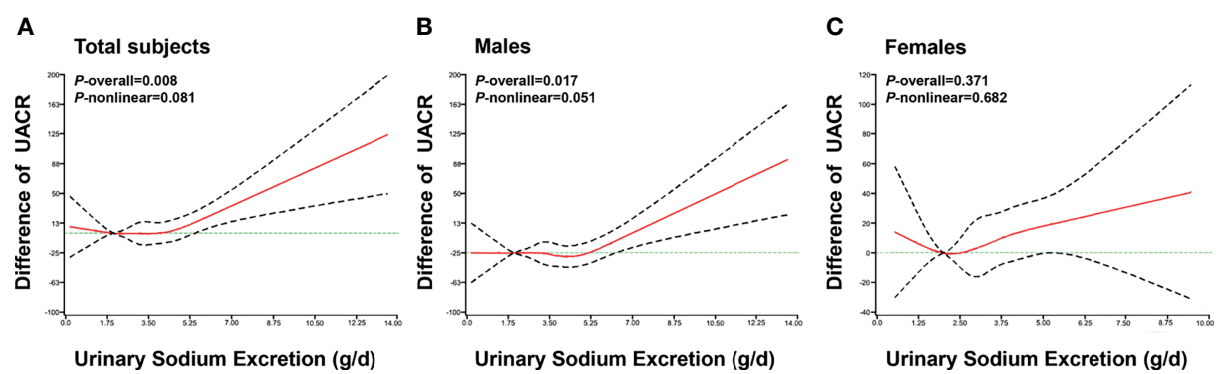

FIGURE 1 | The association of urinary sodium excretion with UACR level. The restricted cubic spline (RCS) regression was used to analyze the relationships of urinary sodium excretion (g/d) with urinary albumin to creatinine ratio (UACR) after adjusting for age, gender, BMI, smoking, alcohol consumption, DBP, RAS blocking agents, diuretics, hyperlipidemia, statin, HbA1c and antidiabetic drugs in total subjects (A), males (B), females (C). Urinary sodium excretion was coded using an RCS function with five knots located at the 5th, 25th, 50th, 75th, 95th percentiles of the distribution of urinary sodium excretion. Y-axis represents the difference in UACR between individuals with any value of urinary sodium excretion with individuals with $2 \mathrm{~g} / \mathrm{d}$ of urinary sodium excretion. $X$-axis represents the continuous change of urinary sodium excretion. Black dashed lines are 95 percent confidence intervals.

in highest quartile of urinary sodium excretion were 1.56 times more likely to have DKD than those in the lowest quartile [OR (95\% CI); 1.56 (1.07-2.27); $P=0.020$ ]; urinary sodium excretion was significantly associated with the increased risks of DKD $(P<0.05)$. However, the relationship between urinary sodium excretion and the risks of DKD showed no significance, after further adjustment for HOMA-IR and PWV $(P>0.05)$. In the subgroup analyses of the associations between the risk of DKD and urinary sodium excretion levels according to the following variables: age ( $<60$ years $/ \geq 60$ years), gender (male/female), hypertension (yes/no), BMI $\left(<24 \mathrm{~kg} / \mathrm{m}^{2 /} \geq 24 \mathrm{~kg} / \mathrm{m}^{2}\right)$, duration of diabetes $(\leq 5$ years $/>5$ years), HbA1c $(<9 \% / \geq 9 \%)$, SGLT_2i (yes/no), Diuretics(yes/no), the result indicated that the relationships of sodium excretion levels and the risk of DKD had no interaction between different subgroups $(P$-interaction $>$ 0.05) (Supplementary Figure 1).

\section{DISCUSSION}

In the present study, we provided the evidence regarding the relationship between sodium intake and the presence of DKD in patients with T2DM. Our data showed that high urinary sodium excretion level was significantly associated with UACR level and increased risk of $\mathrm{DKD}$, and exhibited a J-shaped relationship with UACR level. Of note, the relationship between urinary sodium excretion and risks of DKD became insignificant after further adjustment for HOMA-IR and PWV. These findings indicated that dietary sodium was associated with high risks of DKD among patients with T2DM, dependent of vascular sclerosis and insulin resistance.

Our data showed that patients with DKD had higher levels of urinary sodium excretion level than those without DKD. Prior studies showed that a survey in Japanese illustrated a reverse J-shaped relationship between daily salt intake and albuminuria in patients with T2DM (11). In contrast, Horikawa et al. reported no significant difference between sodium intake and the risk of albuminuria in patients with T2DM (12). A small cross-sectional study reported that a high-sodium diet is an independent influencing factor of microalbuminuria and renal dysfunction in 71 patients with T2DM (21). Of note, those studies used dietary recall to calculate sodium intake or estimated from a spot urine sample, which may cause a recall bias, and did not provide conclusive evidence regarding the relationship between sodium intake and the presence of DKD among the patients with T2DM. On the basis of a relative larger sample size, our study estimated sodium intake by urinary sodium excretion, and indicated that high urinary sodium was significantly associated with the risks of UACR level as well as the presence of DKD in patients with T2DM independent of several traditional risk factors. It has been proposed that high sodium intake can increase concentrations of extracellular sodium, and induce myocardial and renal fibrosis (22). Our findings suggest that monitoring sodium intake might be useful for prevention and treatment of $\mathrm{DKD}$ in patients with T2DM.

Our data indicated that urinary sodium level was independently correlated with UACR level as well as metabolic risk factors, such as BMI, DBP, SBP, HOMA-IR, and PWV. It has been well documented that several metabolic risk factors, including $\mathrm{BMI}$ and blood pressure, play a role in the development of DKD (23-25). Epidemiological study indicated that urinary sodium is linked with incidence of DKD through BMI and blood pressure $(26,27)$. Vedovato et al. reported that a positive correlation between higher sodium intake and albuminuria in obese adults (28). Overweight and obesity were associated with salt sensitivity and even increase glomerular filtration rate (29). Furthermore, Cardoso and colleague reported that elevated blood pressure was the main predictor of development or progression of DKD in patients with T2DM (30). Our study showed that high urinary sodium excretion was associated with the risk of DKD in patients, even adjusting for BP and BMI.

Interestingly, the relationships between urinary sodium excretion and the risks of DKD and albuminuria were no significant after further adjusting for HOMA-IR and PWV in the present study. These findings indicated that the impact of 
TABLE 4 | Odds ratios (ORs) of DKD and albuminuria according to urinary sodium excretion levels.

\begin{tabular}{|c|c|c|c|c|c|c|}
\hline \multirow[t]{2}{*}{ Variables } & \multicolumn{3}{|c|}{ DKD } & \multicolumn{3}{|c|}{ Albuminuria } \\
\hline & OR & $95 \% \mathrm{Cl}$ & $P$-value & OR & $95 \% \mathrm{Cl}$ & $P$-value \\
\hline \multicolumn{7}{|l|}{ Crude model 1} \\
\hline $\begin{array}{l}\text { urinary sodium }(\mathrm{g} / \mathrm{d}) \\
\text { urinary sodium }(\mathrm{g} / \mathrm{d})\end{array}$ & 1.09 & $0.96-1.23$ & 0.189 & 1.19 & $1.02-1.41$ & 0.033 \\
\hline (Quartile 2 vs. Quartile 1) & 0.84 & $0.58-1.22$ & 0.362 & 0.96 & $0.57-1.61$ & 0.871 \\
\hline (Quartile 3 vs. Quartile 1) & 0.96 & $0.67-1.38$ & 0.833 & 0.87 & $0.51-1.48$ & 0.595 \\
\hline (Quartile 4 vs. Quartile 1) & 1.29 & $0.91-1.82$ & 0.154 & 1.17 & $0.71-1.92$ & 0.550 \\
\hline \multicolumn{7}{|l|}{ Crude model 2} \\
\hline $\begin{array}{l}\text { urinary sodium }(\mathrm{g} / \mathrm{d}) \\
\text { urinary sodium }(\mathrm{g} / \mathrm{d})\end{array}$ & 1.12 & $0.99-1.27$ & 0.083 & 1.23 & $1.04-1.46$ & 0.019 \\
\hline (Quartile 2 vs. Quartile 1) & 1.02 & $0.70-1.49$ & 0.905 & 1.23 & $0.70-2.17$ & 0.467 \\
\hline (Quartile 3 vs. Quartile 1) & 1.05 & $0.72-1.52$ & 0.805 & 1.07 & $0.60-1.92$ & 0.809 \\
\hline (Quartile 4 vs. Quartile 1) & 1.44 & $1.00-2.06$ & 0.049 & 1.53 & $0.88-2.65$ & 0.129 \\
\hline \multicolumn{7}{|l|}{ Model 1} \\
\hline $\begin{array}{l}\text { urinary sodium (g/d) } \\
\text { urinary sodium (g/d) }\end{array}$ & 1.13 & $1.00-1.28$ & 0.045 & 1.23 & $1.04-1.45$ & 0.014 \\
\hline (Quartile 2 vs. Quartile 1) & 0.98 & $0.69-1.40$ & 0.906 & 1.09 & $0.65-1.81$ & 0.747 \\
\hline (Quartile 3 vs. Quartile 1) & 1.01 & $0.71-1.45$ & 0.944 & 0.93 & $0.55-1.57$ & 0.781 \\
\hline (Quartile 4 vs. Quartile 1) & 1.42 & $1.01-2.01$ & 0.046 & 1.37 & $0.83-2.26$ & 0.219 \\
\hline \multicolumn{7}{|l|}{ Model 2} \\
\hline $\begin{array}{l}\text { urinary sodium (g/d) } \\
\text { urinary sodium (g/d) }\end{array}$ & 1.19 & $1.04-1.35$ & 0.009 & 1.24 & $1.05-1.47$ & 0.014 \\
\hline (Quartile 2 vs. Quartile 1) & 1.04 & $0.72-1.51$ & 0.839 & 1.10 & $0.65-1.86$ & 0.724 \\
\hline (Quartile 3 vs. Quartile 1) & 1.03 & $0.71-1.50$ & 0.872 & 0.96 & $0.56-1.64$ & 0.878 \\
\hline (Quartile 4 vs. Quartile 1) & 1.62 & $1.13-2.33$ & 0.009 & 1.37 & $0.82-2.31$ & 0.234 \\
\hline \multicolumn{7}{|l|}{ Model 3} \\
\hline $\begin{array}{l}\text { urinary sodium (g/d) } \\
\text { urinary sodium (g/d) }\end{array}$ & 1.17 & $1.03-1.34$ & 0.016 & 1.22 & $1.03-1.46$ & 0.024 \\
\hline (Quartile 2 vs. Quartile 1) & 0.99 & $0.68-1.45$ & 0.976 & 1.03 & $0.61-1.76$ & 0.903 \\
\hline (Quartile 3 vs. Quartile 1) & 1.02 & $0.69-1.49$ & 0.926 & 0.93 & $0.54-1.61$ & 0.800 \\
\hline (Quartile 4 vs. Quartile 1) & 1.56 & $1.07-2.27$ & 0.020 & 1.28 & $0.75-2.17$ & 0.373 \\
\hline \multicolumn{7}{|l|}{ Model 4} \\
\hline $\begin{array}{l}\text { urinary sodium }(\mathrm{g} / \mathrm{d}) \\
\text { urinary sodium }(\mathrm{g} / \mathrm{d})\end{array}$ & 1.13 & $0.99-1.30$ & 0.076 & 1.19 & $0.99-1.43$ & 0.068 \\
\hline (Quartile 2 vs. Quartile 1) & 0.98 & $0.66-1.45$ & 0.914 & 1.11 & $0.62-2.00$ & 0.733 \\
\hline (Quartile 3 vs. Quartile 1) & 0.98 & $0.66-1.47$ & 0.930 & 0.98 & $0.54-1.80$ & 0.959 \\
\hline (Quartile 4 vs. Quartile 1) & 1.45 & $0.98-2.15$ & 0.062 & 1.29 & $0.72-2.33$ & 0.395 \\
\hline \multicolumn{7}{|l|}{ Model 5} \\
\hline $\begin{array}{l}\text { urinary sodium (g/d) } \\
\text { urinary sodium }(\mathrm{g} / \mathrm{d})\end{array}$ & 1.10 & $0.95-1.27$ & 0.208 & 1.17 & $0.97-1.42$ & 0.111 \\
\hline (Quartile 2 vs. Quartile 1) & 0.80 & $0.53-1.22$ & 0.309 & 0.96 & $0.52-1.78$ & 0.900 \\
\hline (Quartile 3 vs. Quartile 1) & 0.93 & $0.61-1.39$ & 0.710 & 0.97 & $0.52-1.80$ & 0.922 \\
\hline (Quartile 4 vs. Quartile 1) & 1.31 & $0.87-1.95$ & 0.194 & 1.51 & $0.63-2.11$ & 0.649 \\
\hline
\end{tabular}

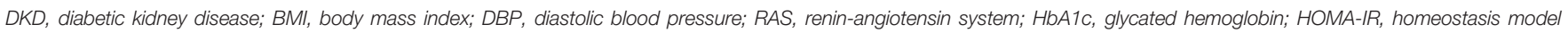
assessment of insulin resistance; PWW, pulse wave velocity.

Crude model 1: adjusted for HOMA-IR.

Crude model 2: adjusted for PWV.

Model 1: adjusted for age, gender, BMI, smoking and alcohol consumption.

Model 2: adjusted for model 1+DBP, RAS blocking agents, diuretics, hyperlipidemia and statin.

Model 3: adjusted for model 2+ HbA1C and antidiabetic medication.

Model 4: adjusted for model 3+ PWV.

Model 5: adjusted for model 4+ HOMA-IR.

urinary sodium excretion on DKD may be mediated by other mechanisms, such as vascular sclerosis or insulin resistance. Observational study in Japanese found that individuals with increased PWV was associated with an increased incidence of albuminuria and reduced renal function (31). The proposed mechanisms between arterial stiffness and albuminuria may be involved that increased pulsatile stress from the stiffening of large arteries elevated intrarenal pulse pressure, and lead to microvascular damage and renal insufficiency (32). In addition, insulin resistance is closely correlated with endothelial dysfunction, mild inflammation and oxidative stress, which is involved in the development of arteriosclerosis and DKD (33). Thus, our finding suggests that high urinary sodium excretion is linked with the risks of DKD through increased arteriosclerosis and insulin resistance.

Additionally, our data indicated that $2 \mathrm{~g} / \mathrm{d}$ of sodium excretion were associated with lower UACR levels, which is consistent with the recommendation by WHO (20). Recently, 
ADA also recommended a reduction to $<2 \mathrm{~g} / \mathrm{d}$ sodium $(5 \mathrm{~g} / \mathrm{d}$ salt) in patients with diabetes (34). However, guidelines from UK and USA recommended sodium intake reduction that is based on the relationship between high sodium intake and the risks of hypertension and $\operatorname{CVD}(35,36)$. Previous study reported that lower 24 -h urinary sodium excretion $(<150 \mathrm{mmol} \mathrm{Na}$ /day) was inversely associated with increased all-cause mortality (37), which may be higher than the recommended intake $(5 \mathrm{~g} / \mathrm{d}$ salt). Thomas et al. showed that the lowest sodium excretion was associated with the highest cumulative incidence of ESRD in the subgroup of 424 patients with macroalbuminuria (38). Additionally, this study also showed that both high and low sodium intake were associated with adverse mortality outcomes. Our study illustrated that there was a J-shaped relationship between sodium intake and UACR level in patients with T2DM rather than monotonic linear relations. This result also suggested that both higher and lower sodium intake were associated with increased urinary albumin excretion and might cause damage to renal function, underscoring the importance of moderate restriction of sodium intake for reducing the risk of DKD.

This study has the following limitations. First, the study was a cross-sectional design. Causality between urinary sodium excretion and the presence of DKD cannot be determined. It is necessary to determine the association of sodium intake and renal outcomes with long term follow-up period in the prospective cohort study. Second, we used morning fasting urine samples instead of $24 \mathrm{~h}$ urine samples for estimating sodium excretion in outpatients.

\section{CONCLUSION}

In conclusion, our data demonstrated that high urinary sodium excretion was associated with high risk of DKD among patients with T2DM, dependent of vascular sclerosis and insulin resistance. Our findings suggest that moderate restriction of sodium intake might be benefit for reducing the risk of DKD. Further study needs to determine the association of sodium intake and the presence of DKD in the prospective studies.

\section{DATA AVAILABILITY STATEMENT}

The raw data supporting the conclusions of this article will be made available by the authors, without undue reservation.

\section{REFERENCES}

1. Sabanayagam C, Chee ML, Banu R, Cheng CY, Lim SC, Tai ES, et al. Association of Diabetic Retinopathy and Diabetic Kidney Disease With AllCause and Cardiovascular Mortality in a Multiethnic Asian Population. JAMA Netw Open (2019) 2(3):e191540. doi: 10.1001/jamanetworkopen

2. Tuttle KR, Bakris GL, Bilous RW, Chiang JL, de Boer IH, Goldstein-Fuchs J, et al. Diabetic Kidney Disease: A Report From an ADA Consensus Conference. Am J Kidney Dis (2014) 64(4):510-33. doi: 10.1053/j.ajkd.2014.08.001

\section{ETHICS STATEMENT}

The study protocol was approved by the Institutional Review Board of Nanfang Hospital of Southern Medical University. The patients/participants provided their written informed consent to participate in this study.

\section{AUTHOR CONTRIBUTIONS}

$\mathrm{YH}, \mathrm{HZ}$, and YX contributed to conception and design of the study. HZ and YX supervised the study. DG, PZ, DL and LY organized the database. YH, JL and Jy L performed the statistical analysis. YH wrote the first draft of the manuscript. YH, WL and JL wrote sections of the manuscript. All authors contributed to the article and approved the submitted version.

\section{FUNDING}

The National Key Research and Development Project (No.2018YFA0800404); Natural Science Foundation and KeyArea Research and Development Program of Guangdong Province (No.2018B030311031 and 2019B020227004); Clinical Research Startup Program of Southern Medical University by High-level University Construction Funding of Guangdong Provincial Department of Education (No.LC2019ZD010 and 2019CR022); the Medical Scientific Research Foundation of Guangdong Province (No. A2019504); President Foundation of Nanfang Hospital, Southern Medical University (No. 2018C028).

\section{ACKNOWLEDGMENTS}

The authors are grateful to all participants for their time and effort.

\section{SUPPLEMENTARY MATERIAL}

The Supplementary Material for this article can be found online at: https://www.frontiersin.org/articles/10.3389/fendo.2021. 772073/full\#supplementary-material

Supplementary Figure 1 | Odds ratios of DKD in different subgroups. Forest plot of odds ratios of DKD according to urinary sodium excretion levels in different subgroups. Patients were categorized according to the following variables: age ( $<60$ years/ $>60$ years), gender (male/female), hypertension (yes/no), BMl $(<24 \mathrm{~kg} /$ $\mathrm{m}^{2} / \geq 24 \mathrm{~kg} / \mathrm{m}^{2}$ ), duration of diabetes ( $\leq 5$ years $/>5$ years), $\mathrm{HbA1c}(<9 \% / \geq 9 \%)$, SGLT_2i(yes/no), Diuretics(yes/no).

3. Morrish NJ, Wang SL, Stevens LK, Fuller JH, Keen H. Mortality and Causes of Death in the WHO Multinational Study of Vascular Disease in Diabetes. Diabetologia (2001) 44(Suppl 2):S14-21. doi: 10.1007/pl00002934

4. Xu Y, Wang L, He J, Bi Y, Li M, Wang T, et al. Prevalence and Control of Diabetes in Chinese Adults. JAMA (2013) 310(9):948-59. doi: 10.1001/ jama.2013.168118

5. Mills KT, Chen J, Yang W, Appel LJ, Kusek JW, Alper A, et al. Sodium Excretion and the Risk of Cardiovascular Disease in Patients With Chronic Kidney Disease. JAMA (2016) 315(20):2200-10. doi: 10.1001/jama.2016.4447 
6. He J, Mills KT, Appel LJ, Yang W, Chen J, Lee BT, et al. Urinary Sodium and Potassium Excretion and CKD Progression. J Am Soc Nephrol (2016) 27 (4):1202-12. doi: 10.1681/ASN.2015010022

7. Remuzzi G, Schieppati A, Ruggenenti P. Clinical Practice. Nephropathy in Patients With Type 2 Diabetes. N Engl J Med (2002) 346(15):1145-51. doi: 10.1056/NEJMcp011773

8. Viberti GC, Hill RD, Jarrett RJ, Argyropoulos A, Mahmud U, Keen H. Microalbuminuria as a Predictor of Clinical Nephropathy in InsulinDependent Diabetes Mellitus. Lancet (1982) 1(8287):1430-2. doi: 10.1016/ s0140-6736(82)92450-3

9. Engelen L, Soedamah-Muthu SS, Geleijnse JM, Toeller M, Chaturvedi N, Fuller JH, et al. Higher Dietary Salt Intake is Associated With Microalbuminuria, But Not With Retinopathy in Individuals With Type 1 Diabetes: The EURODIAB Prospective Complications Study. Diabetologia (2014) 57(11):2315-23. doi: 10.1007/s00125-014-3367-9

10. Verhave JC, Hillege HL, Burgerhof JG, Janssen WM, Gansevoort RT, Navis GJ, et al. Sodium Intake Affects Urinary Albumin Excretion Especially in Overweight Subjects. J Intern Med (2004) 256(4):324-30. doi: 10.1111/j.13652796.2004.01390.x

11. Sakabe K, Fukui M, Ushigome E, Hamaguchi M, Senmaru T, Yamazaki M, et al. Low Daily Salt Intake is Correlated With Albuminuria in Patients With Type 2 Diabetes. Hypertens Res (2012) 35(12):1176-9. doi: 10.1038/hr.2012.116

12. Horikawa C, Yoshimura Y, Kamada C, Tanaka S, Tanaka S, Hanyu O, et al. Dietary Sodium Intake and Incidence of Diabetes Complications in Japanese Patients With Type 2 Diabetes: Analysis of the Japan Diabetes Complications Study (JDCS). J Clin Endocrinol Metab (2014) 99(10):3635-43. doi: 10.1210/ jc. $2013-4315$

13. Luft FC, Fineberg NS, Sloan RS. Estimating Dietary Sodium Intake in Individuals Receiving a Randomly Fluctuating Intake. Hypertension (1982) 4(6):805-8. doi: 10.1161/01.hyp.4.6.805

14. Liu J, Guo D, Zhang P, Yang X, Lin J, Liu D, et al. Association of Urinary Sodium Excretion and Left Ventricular Hypertrophy in Patients With Type 2 Diabetes: A Cross-Section Study, PREPRINT (Version 1) (2021). Available at: https://doi.org/10.21203/rs.3.rs-239931/v1.

15. American Diabetes Association. 2. Classification and Diagnosis of Diabetes. Diabetes Care (2017) 40(Suppl 1):S11-24. doi: 10.2337/dc17-S005

16. Kawasaki T, Itoh K, Uezono K, Sasaki H. A Simple Method for Estimating 24 H Urinary Sodium and Potassium Excretion From Second Morning Voiding Urine Specimen in Adults. Clin Exp Pharmacol Physiol (1993) 20(1):7-14. doi: 10.1111/j.1440-1681.1993.tb01496.x

17. Kawamura M, Kusano Y, Takahashi T, Owada M, Sugawara T. Effectiveness of a Spot Urine Method in Evaluating Daily Salt Intake in Hypertensive Patients Taking Oral Antihypertensive Drugs. Hypertens Res (2006) 29 (6):397-402. doi: 10.1291/hypres.29.397

18. Levey AS, Stevens LA, Schmid CH, Zhang YL, Castro AF3rd, Feldman HI, et al. A New Equation to Estimate Glomerular Filtration Rate. Ann Intern Med (2009) 150(9):604-12. doi: 10.7326/0003-4819-150-9-200905050-00006

19. American Diabetes Association. 10. Microvascular Complications and Foot Care. Diabetes Care (2017) 40(Suppl 1):S88-98. doi: 10.2337/dc17-S013

20. WHO Guidelines Approved by the Guidelines Review Committee. Guideline: Sodium Intake for Adults and Children. Geneva: World Health Organization (2012).

21. Kawabata N, Kawamura T, Utsunomiya K, Kusano E. High Salt Intake Is Associated With Renal Involvement in Japanese Patients With Type 2 Diabetes Mellitus. Intern Med (2015) 54(3):311-7. doi: 10.2169/ internalmedicine.54.2464

22. Yu HC, Burrell LM, Black MJ, Wu LL, Dilley RJ, Cooper ME, et al. Salt Induces Myocardial and Renal Fibrosis in Normotensive and Hypertensive Rats. Circulation (1998) 98(23):2621-8. doi: 10.1161/01.cir.98.23.2621

23. Todd JN, Dahlström EH, Salem RM, Sandholm N, Forsblom CFinnDiane Study Group, et al. Genetic Evidence for a Causal Role of Obesity in Diabetic Kidney Disease. Diabetes (2015) 64(12):4238-46. doi: 10.2337/db15-0254

24. Patel DM, Bose M, Cooper ME. Glucose and Blood Pressure-Dependent Pathways-The Progression of Diabetic Kidney Disease. Int J Mol Sci (2020) 21 (6):2218. doi: 10.3390/ijms21062218

25. Duan J, Wang C, Liu D, Qiao Y, Pan S, Jiang D, et al. Prevalence and Risk Factors of Chronic Kidney Disease and Diabetic Kidney Disease in Chinese
Rural Residents: A Cross-Sectional Survey. Sci Rep (2019) 9(1):10408. doi: 10.1038/s41598-019-46857-7

26. Man REK, Gan ATL, Fenwick EK, Gupta P, Wong MYZ, Wong TY, et al. The Relationship Between Generalized and Abdominal Obesity With Diabetic Kidney Disease in Type 2 Diabetes: A Multiethnic Asian Study and MetaAnalysis. Nutrients (2018) 10(11):1685. doi: 10.3390/nu10111685

27. De Cosmo S, Viazzi F, Piscitelli P, Giorda C, Ceriello A, Genovese S, et al. Blood Pressure Status and the Incidence of Diabetic Kidney Disease in Patients With Hypertension and Type 2 Diabetes. J Hypertens (2016) 34 (10):2090-8. doi: 10.1097/HJH.0000000000001045

28. Aaron KJ, Campbell RC, Judd SE, Sanders PW, Muntner P. Association of Dietary Sodium and Potassium Intakes With Albuminuria in Normal-Weight, Overweight, and Obese Participants in the Reasons for Geographic and Racial Differences in Stroke (REGARDS) Study. Am J Clin Nut (2011) 94(4):1071-8. doi: 10.3945/ajen.111.013094

29. Ribstein J, du Cailar G, Mimran A. Combined Renal Effects of Overweight and Hypertension. Hypertension (1995) 26(4):610-5. doi: 10.1161/01.hyp.26.4.610

30. Cardoso CRL, Leite NC, Salles GC, Ferreira MT, Salles GF. Aortic Stiffness and Ambulatory Blood Pressure as Predictors of Diabetic Kidney Disease: A Competing Risks Analysis From the Rio De Janeiro Type 2 Diabetes Cohort Study. Diabetologia (2018) 61(2):455-65. doi: 10.1007/s00125-017-4484-z

31. Bouchi R, Babazono T, Mugishima M, Yoshida N, Nyumura I, Toya K, et al. Arterial Stiffness is Associated With Incident Albuminuria and Decreased Glomerular Filtration Rate in Type 2 Diabetic Patients. Diabetes Care (2011) 34(12):2570-5. doi: $10.2337 / \mathrm{dc} 11-1020$

32. O'Rourke MF, Safar ME. Relationship Between Aortic Stiffening and Microvascular Disease in Brain and Kidney: Cause and Logic of Therapy. Hypertension (2005) 46(1):200-4. doi: 10.1161/01.HYP.0000168052.00426.65

33. Karalliedde J, Gnudi L. Diabetes Mellitus, a Complex and Heterogeneous Disease, and the Role of Insulin Resistance as a Determinant of Diabetic Kidney Disease. Nephrol Dial Transplant (2016) 31(2):206-13. doi: 10.1093/ ndt/gfu405

34. American Diabetes Association, Bantle JP, Wylie-Rosett J, Clark NG, Franz MJ, Hoogwerf BJ, et al. Nutrition Recommendations and Interventions for Diabetes: A Position Statement of the American Diabetes Association. Diabetes Care (2008) 31(Suppl 1):S61-78. doi: 10.2337/dc08-S061

35. Dyson PA, Twenefour D, Breen C, Duncan A, Elvin E, Goff L, et al. Diabetes UK Evidence-Based Nutrition Guidelines for the Prevention and Management of Diabetes. Diabetes Med (2018) 35(5):541-7. doi: 10.1111/dme.13603

36. Evert AB, Boucher JL, Cypress M, Dunbar SA, Franz MJ, Mayer-Davis EJ, et al. Nutrition Therapy Recommendations for the Management of Adults With Diabetes. Diabetes Care (2014) 37 Suppl 1:S120-43. doi: 10.2337/dc14-S120

37. Ekinci EI, Clarke S, Thomas MC, Moran JL, Cheong K, MacIsaac RJ, et al. Dietary Salt Intake and Mortality in Patients With Type 2 Diabetes. Diabetes Care (2011) 34(3):703-9. doi: 10.2337/dc10-1723

38. Thomas MC, Moran J, Forsblom C, Harjutsalo V, Thorn L, Ahola A, et al. The Association Between Dietary Sodium Intake, ESRD, and All-Cause Mortality in Patients With Type 1 Diabetes. Diabetes Care (2011) 34(4):861-6. doi: $10.2337 / \mathrm{dc} 10-1722$

Conflict of Interest: The authors declare that the research was conducted in the absence of any commercial or financial relationships that could be construed as a potential conflict of interest.

Publisher's Note: All claims expressed in this article are solely those of the authors and do not necessarily represent those of their affiliated organizations, or those of the publisher, the editors and the reviewers. Any product that may be evaluated in this article, or claim that may be made by its manufacturer, is not guaranteed or endorsed by the publisher.

Copyright (๑) 2021 Huang, Liu, Liu, Guo, Zhang, Liu, Lin, Yang, Zhang and Xue. This is an open-access article distributed under the terms of the Creative Commons Attribution License (CC BY). The use, distribution or reproduction in other forums is permitted, provided the original author(s) and the copyright owner(s) are credited and that the original publication in this journal is cited, in accordance with accepted academic practice. No use, distribution or reproduction is permitted which does not comply with these terms. 